\title{
Routing optimization method of waste transportation vehicle using biological evolutionary algorithm under the perspective of low carbon and environmental protection
}

\author{
Linsheng $\operatorname{Fan}^{1,2+}$ \\ ${ }^{I} X i$ 'an Jiaotong University, Xi'an, Shaanxi, 710049, China \\ ${ }^{2}$ Guangxi Chongzuo Urban Construction Investment Development Group Co., Ltd., Chongzuo, Guangxi, 532200, China
}

Received September 18,2021 Revised November 09,2021 Accepted December 27, 2021

\begin{abstract}
Reasonably and effectively formulating the best route for urban waste transportation vehicle is particularly important for realizing low carbon and environmental protection of Green China construction concept. However, the current path planning method has shortcomings such as local optimization. In order to solve this problem, this paper aims at low carbon and environmental protection construction needs and proposes a routing optimization method of waste transportation vehicles based on improved ant colony algorithm. Firstly, the mathematical model of Vehicle Routing Problem (VRP) is constructed by considering transportation distance and carbon emissions cost. Then, network parameters in traditional ant colony algorithm are combined and optimized to realize self-adaptive update in the whole cycle. Furthermore, the neighborhood algorithm is used to iteratively optimize improved algorithm to avoid the defects of local optimization when solving VRP problem. Finally, the simulation results based on an actual dataset in North China show that the proposed method can achieve efficient and accurate optimal routing optimization for complex samples, and its solution stability index $P_{\text {best }}$ is 0.87 and the average deviation $V_{a r}$ is 0.011 , the lowest distribution cost after optimization is about 3,080 yuan, which are better than the comparison methods.
\end{abstract}

Keywords: Ant colony algorithm, Carbon emission, Neighborhood algorithm, Parameter optimization, Pheromone update, Routing optimization

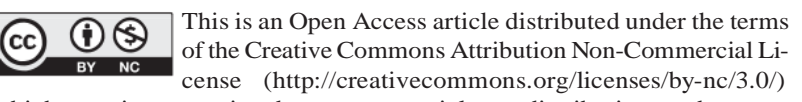
which permits unrestricted non-commercial use, distribution, and reproduction in any medium, provided the original work is properly cited.

\author{
Corresponding Author \\ E-mail: fanlinsheng2021@163.com \\ Tel: +18077186541
}

ORCID: 0000-0001-5040-3121 


\section{Introduction}

2 With the rapid development of social economy and acceleration of urbanization, the amount of

3 various types for waste has increased sharply [1-3]. According to incomplete statistics, the total

4 amount of domestic waste in China's cities and towns ranks first in the world. In recent years,

5 with the acceleration of my country's urbanization process and the improvement of people's

6 living standards, urban domestic waste is still increasing at an annual rate of 5 to $8 \%$ [4].

At present, on the one hand, the amount of waste is huge, on the other hand, there are phenomena such as backward transportation management methods and high equipment operation

9 costs in the process of waste collection and transportation [5, 6]. This will lead to excessive 10 waste of resources and low work efficiency problems in the current waste transportation links.

According to statistics, $80-85 \%$ of waste disposal system cost is attributed to the

12 transportation system [7]. At the same time, the carbon dioxide emissions of my country's transportation industry are showing an increasing trend, and transportation energy consumption

14 accounts for about $9 \%$ of the total energy consumption of whole society [8]. With the transfer of 15 waste treatment facilities to the outer suburbs and expansion of main urban area, the coverage of 16 entire waste collection and transportation system is getting larger and larger. Therefore, scientific 17 and reasonable planning of waste collection and transportation routes is an important guarantee 18 for the realization for concept of "low energy consumption, low pollution and low emissions", 19 which is particularly urgent.

In recent years, the academic research on waste collection path is mainly based on the 21 corresponding analysis of Traveling Salesman Problem (TSP) and the Chinese Postman Problem 22 (CPP) $[9,10]$. But in fact, we can divide waste collection and transportation routes into two 
1 aspects: waste collection routes and waste transfer routes. If focusing on the two aspects of

2 modeling ideas and solving goals, the waste collection routing problem should belong to the 3 category of Vehicle Routing Problem (VRP) [11, 12].

4 At present, some researchers have discussed this based on intelligent algorithms. 5 Reference [13] established cost-oriented urban distribution optimization model, adopted an 6 improved genetic algorithm and designed successive correction operators on both sides to 7 improve the solution quality and efficiency of algorithm. Reference [14] established mixed 8 integer programming model and designed a joint optimization genetic algorithm to solve the

9 model. It used the proposed joint optimization genetic algorithm and two-stage genetic algorithm 10 for simulation calculation and analysis. Reference [15] proposed a hybrid method based on sine 11 cosine algorithm and paper swarm algorithm to solve the problem of heterogeneous vehicle 12 routing. Reference [16] was based on the gray wolf spatial integer coding and solution of routing 13 before grouping. It proposed an adaptive genetic gray wolf optimization algorithm to solve VRP 14 with capacity constraints. However, it should be noted that the current routing optimization 15 methods based on intelligent algorithms generally have the problems of weak adaptive ability 16 and weak global optimization convergence ability [17]. This make the problem of searching for 17 the best path in complex space often lose the optimal solution, and it will cause network to 18 diverge in severe cases.

19 Aiming at the problems existing in current VRP planning method, this paper proposes a 20 routing optimization method for waste transportation vehicles that meets the needs of low carbon 21 environmental protection based on improved ant colony algorithm. The innovative points of 22 proposed method are: 
1) In order to further improve the optimization characteristics of the best route for waste

2 vehicle transportation, this paper optimizes the network parameters based on traditional ant 3 colony algorithm, which improves the global convergence and solution efficiency of the ant 4 colony algorithm.

5 2) The pheromone setting method in the network is improved to realize the full-period self6 adaptive update of network parameters and avoids losing the global optimal solution.

7 The remaining content of this paper is arranged as follows: Section 2 describes and models

8 VRP problem accordingly. Section 3 describes the corresponding improvement content of ant 9 colony algorithm. Section 4 is the simulation verification of proposed method based on actual sample dataset. Section 5 is the conclusion of this paper.

\section{2. Problem Description and Model Construction}

\section{2.1. Problem Description}

14 Waste management requires not only government investment and management, but also 15 residents' awareness of environmental protection. The government needs to increase waste 16 collection facilities in each community under its jurisdiction and arrange waste vehicles to 17 recycle waste in each community in time.

In an area, the process of waste collection can be described as follows: multiple waste 19 vehicles depart from parking lot at the same time and begin to collect waste in various 20 communities. Each community must be served and can only be served once (assuming that the 21 amount of waste generated in each community is less than or equal to the maximum load 22 capacity of waste vehicles). When the waste vehicle reaches or is close to the maximum load, it 
1 will first drive to transfer stations, unload the waste and then return to the depot. The goal of 2 solving the problem is to find a shortest path that minimizes the total distance traveled by all 3 waste vehicles. Fig. 1 is a simple schematic diagram of waste collection path.

4

6

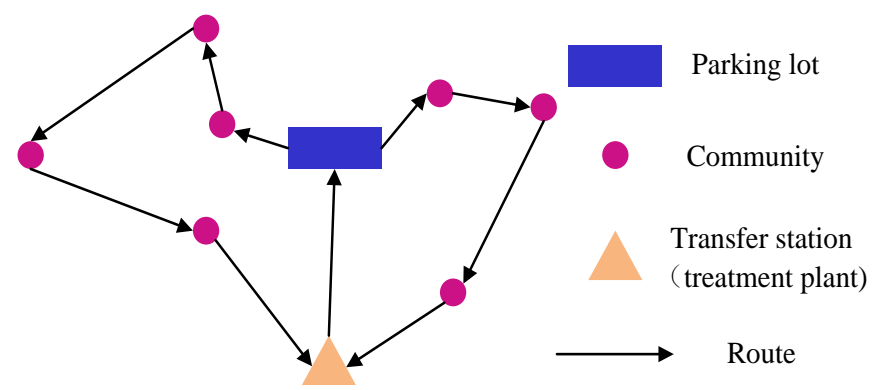

Fig. 1. Schematic diagram of waste vehicle path.

As can be seen in Fig. 1, there are a total of 7 communities, and 2 waste vehicles can be used to complete the waste collection work in the community. Each waste vehicle departs from parking lot and starts to serve the community. After the waste collection volume reaches or approaches the maximum load capacity, waste vehicles go to the transfer station (treatment plant) to unload waste. Finally it returns to the parking lot.

In order to better describe the mathematical model of problem, the model elements are elaborated accordingly. The components of waste collection path problem include: parking lot, waste vehicle, waste, community, transfer station (processing plant), constraint conditions and objective function.

\subsubsection{Depot}

The parking lot is similar to logistics distribution center. It is the starting point and ending point of the waste vehicle's driving process. According to the number of parking lots, it can be divided 
1 into single parking lot and multiple parking lot. However, considering the actual situation,

2 usually a parking lot in an area can park required waste vehicles. So this paper studies the 3 situation of bicycle yard.

4

$5 \quad$ 2.1.2. Waste vehicle

6 Waste vehicle is a tool for transporting waste, which can be divided into single vehicle type and 7 multiple vehicle types. The difference of model often determines the difference in maximum

8 load. The maximum load capacity refers to the upper limit of waste vehicle loading capacity. The 9 type of waste loaded is divided into solid and non-solid. The load condition refers to full load or 10 partial load. The number of vehicles refers to the number of waste vehicles, which are divided 11 into single vehicles and multiple vehicles according to their differences. The maximum mileage 12 limit refers to whether to consider the restriction of waste vehicle mileage [18]. This paper 13 mainly studies the problem of recycling path of community domestic waste. Most domestic 14 waste is solid waste, so a waste vehicle loaded with solid waste is selected for research. 15 Considering comprehensively, multiple waste vehicles of the same model (the same maximum 16 load) are used for waste collection. The waste vehicles are fully loaded and mileage limit is not 17 considered.

19 2.1.3. Waste

20 Waste is similar to goods in logistics transportation, with attributes such as weight and collection 21 location. These two attributes are the basis for waste vehicles to choose the driving route. 
2 Each community is a service object corresponding to a waste collection location, and must be 3 served once.

4

$5 \quad$ 2.1.5. Transfer station (processing plant)

6 Taking into account the actual situation of community waste treatment by county, this element 7 may be a transfer station or a treatment plant. According to their different numbers, it can be 8 divided into single transfer station (treatment plant) and multiple transfer station (treatment 9 plant). In order to reduce the complexity of solving the problem, this paper conducts research on 10 the premise of a single transfer station (processing plant).

$12 \quad$ 2.1.6. Constraint conditions

13 Waste vehicles must meet the following constraints when reclaiming waste: (1) Each waste 14 vehicle must start from the yard; (2) The loading capacity is close to but not exceeding its own 15 maximum load; (3) It must be transported before returning to the yard. The station (treatment 16 plant) unloads the waste; (4) Every community must be served and only served once.

17 The time for waste collection by waste vehicles is arranged by the government every day, and it 18 will not be shifted by the will of a certain community. There is no strong internal relationship 19 between service and time, and the time window constraint has no practical significance in 20 solving this problem. Thus, this paper does not consider the time window limitation.

21

22 2.1.7. Objective function 
1 The commonly used objective functions for solving VRP problems are: (1) The total mileage of

2 vehicles is the shortest; (2) The time-consuming is the least; (3) The cost is the least. This paper

3 uses objective function (1) when solving the waste collection path problem, that is, the sum of all

$4 \quad$ waste vehicles has the shortest mileage.

\section{2.2. Model Construction}

7 The routing optimization problem of waste transportation vehicles can be represented by an

8 undirected graph $G=(V, E)$ [19]. For ease of expression, the following symbols are introduced:

9 point set $V=\{1,2, \ldots \ldots, n\}$ of parking lot, community, transfer station (processing plant), where

10 point $i=1$ represents parking lot, point $i=n$ represents transfer station (processing plant), the

11 remaining points represent communities, and the number of communities is $n-2$. The element

12 in edge set $E$ is $d_{i j}$, which represents the distance between point $i$ and point $j$. The point may

13 be a parking lot, a community or a transfer station (processing plant). The waste discharge of

14 each community is $w_{i}(i=2,3, \ldots \ldots, n-1)$. There are $r(k=1,2, \ldots \ldots, r)$ waste vehicles for waste

15 collection in these $n-2$ communities. Each vehicle has the same model, and the maximum load

16 capacity is $W$ and $W \geq w_{i}(i=2,3, \ldots \ldots, n-1)$. Define the following variables:

$$
x_{i j}{ }^{k}= \begin{cases}1, & \text { the garbage truck } k \text { runs from } i \text { to } j \\ 0, & \text { otherwise }\end{cases}
$$

19 all waste vehicles is taken to the minimum.

$$
Z=\min \sum_{k=1}^{r} \sum_{i, j=1, i \neq j}^{n} d_{i j} x_{i j}{ }^{k}
$$


1 The constraints that the model should meet are as follows:

$$
x_{n j}{ }^{k}=\left\{\begin{array}{ll}
1, & j=1 \\
0, & \text { otherwise }
\end{array}, k \in\{1,2, \ldots \ldots, r\}, j \in V\right.
$$$$
x_{i 1}{ }^{k}=\left\{\begin{array}{ll}
1, & i=n \\
0, & \text { otherwise }
\end{array}, k \in\{1,2, \ldots \ldots, r\}, i \in V\right.
$$

$$
\sum_{i=2}^{n-1} \sum_{j=2, i \neq j}^{n-1} w_{i} x_{i j}{ }^{k} \leq W, k \in\{1,2, \ldots \ldots, r\}
$$

In the above constraints, Eq. (3) indicates that waste vehicles must and can only return to the depot after arriving at transfer station (processing plant). Eq. (4) means that waste vehicles must arrive at the transfer station (processing plant) to unload the waste before returning to the parking lot. Eq. (5) means that every waste vehicle must depart from the parking lot and drive to transfer station (processing plant) after completing tasks. From the first two constraints, the driving route of each waste vehicle can be obtained as: parking lot-each community node-transfer station (processing plant)-parking lot. Eq. (6) and (7) indicate that every community can be served and can only be served once. Eq. (8) indicates that the total amount of waste collected by each waste vehicle in the community must not exceed its own maximum load.

However, a large amount of carbon emissions will be generated in the process of waste transportation, which will not only pollute the environment, but also increase the transportation 
1 cost of responsible department. Therefore, we also introduce carbon emissions into the routing 2 optimization analysis.

The cost of carbon emissions mainly refers to the cost of carbon emissions generated in

4 the transportation of waste vehicles. Carbon emissions are the product of fuel consumption and

$5 \mathrm{CO} 2$ emission coefficient. The fuel consumption of vehicles is related to the load capacity and

6 driving distance of vehicles. The relationship between the fuel consumption per unit distance of a

7 transport vehicle and the load capacity can be expressed as:

$$
\kappa\left(w_{i}\right)=\rho_{0}+\frac{\rho^{*}-\rho_{0}}{W} w_{i}
$$

9 Among them, ${ }^{\rho_{0}}$ represents the fuel consumption per unit distance when the transport vehicle is 10 empty; $\rho^{*}$ represents the fuel consumption per unit distance when the transport vehicle is fully 11 loaded; $W$ represents the full load capacity of vehicles. Then, the target formula of routing optimization problem of waste transportation vehicles

13 from the perspective of low carbon environmental protection is rewritten as:

$$
\begin{aligned}
G & =\min \xi \varepsilon \kappa\left(w_{i}\right) Z \\
& =\min \sum_{k=1}^{r} \sum_{i, j=1, i \neq j}^{n} \xi \varepsilon d_{i j} x_{i j}^{k} \kappa\left(w_{i}\right)
\end{aligned}
$$

15 where ${ }^{\xi}$ represents the carbon tax; $\varepsilon$ represents the carbon emission coefficient. The community waste collection routing problem belongs to the category of VRP 17 problem, which is similar to Capacitated VRP (CVRP) [20, 21]. Compared with the CVRP 18 model, the biggest difference in the mathematical model of this paper is that each waste vehicle 19 must drive to the transfer station (treatment plant) to unload waste before returning to the depot, 20 and then return from the transfer station (treatment plant) to depot. 


\section{3. Routing Optimization of Waste Transportation Vehicles Based on}

\section{Improved Ant Colony Algorithm}

3 Ant colony algorithm has a certain memory function, which can continuously transfer from an

4 old state to a new state when solving combinatorial optimization problems. But there are defects

5 of local optimization and low search ability [22, 23]. For this reason, this paper improves

6 traditional ant colony algorithm to realize the model solution of waste transportation vehicle

7 routing optimization.

8

\section{3.1. Mathematical Model of Ant Colony Algorithm}

10 Before ant colony algorithm completes an iteration, the ants can only visit each node once, and 11 return to the initial node after visiting all nodes. Ants secrete pheromone along the path they 12 traveled. The amount and time of pheromone secretion are determined by algorithm. Assuming that the number of communities to be visited is ${ }^{n}$, the size of ant colony is ${ }^{m}$, 14 and the distance between nodes $i$ and $j$ is $d_{i j}$, then $d_{i j}=\sqrt{\left(x_{i}-x_{j}\right)^{2}+\left(y_{i}-y_{j}\right)^{2}}$, where ${ }^{x_{i}}, x_{j}, y_{i}$ and ${ }^{y_{j}}$ represent the horizontal and vertical coordinates of nodes ${ }^{i}$ and ${ }^{j}$ respectively or latitude and longitude coordinates. At the beginning of each iteration, ${ }^{m}$ ants will be randomly placed in

17 these ${ }^{n}$ nodes, making the starting point of ants random. In order to prevent the same ant from accessing the same node multiple times in one iteration, we set up a taboo table to record nodes that each ant has visited. ${ }^{t a b u_{k}}$ records the names and order of the nodes that ant ${ }^{k}$ have visited. $20 \tau_{i j}(t)$ represents the pheromone concentration on the path ${ }^{<i, j\rangle}$ at time ${ }^{t}$. At the initial time (at

21 time $t=0$ ), the pheromone concentration on all paths is set to the same constant. The probability 
1 that ant ${ }^{k}$ moves from current node $i$ to node ${ }^{j}$ at time $t$ is represented by ${ }^{p_{i j}}{ }^{k}(t)$. We call this

2 state transition rule a random proportional rule, and its formula is as follows:

$$
p_{i j}{ }^{k}(t)= \begin{cases}\frac{\left[\tau_{i j}(t)\right]^{\alpha}\left[\eta_{i j}\right]^{\beta}}{\sum_{s \in \text { allowed }_{k}}\left[\tau_{i s}(t)\right]^{\alpha}\left[\eta_{i s}\right]^{\beta}} & j \in \text { allowed }_{k} \\ 0 & \text { otherwise }\end{cases}
$$

4 where the heuristic factor $\eta_{i j}$ represents the visibility of path $\langle i, j\rangle$, which reflects the heuristic 5 degree of ants moving from node $i$ to ${ }^{j}$, usually its value is:

$$
\eta_{i j}=\frac{1}{d_{i j}}
$$

7 Parameter $\alpha$ and parameter $\beta$ respectively reflect the relative importance of pheromone 8 concentration and heuristic information on the path when ants choose the path. The value of $\alpha$ 9 affects the ant's choice of repeated paths. The larger the value, the greater the ant's ability to walk, 10 which will make the algorithm prone to stagnation. The node numbers that ants have not yet 11 visited are stored in allowed $_{k}$, and the calculation formula is as follows.

$$
\text { allowed }_{k}=\{1,2, \ldots \ldots, n\}-\text { tabu }_{k}
$$

13 When ant ${ }^{k}$ selects an accessible node ${ }^{c}$ according to the random ratio strategy and arrives, we 14 need to update the taboo table, that is, add the number of node $c$ to $t a b u_{k}$. If $\left|t a b u_{k}\right|<n$, then ant $15 k$ continues to select the next node and visit according to the random ratio strategy. After 16 repeating the above process, until ${ }^{t a b u_{k}}$ contains all the communities. At this time, ant $k$ returns 17 to the starting point and adds starting community node to the end of ${ }^{t a b u_{k}}$. When $m$ ants have 18

$$
\text { completed their visit tasks, calculate the total distance traveled by each ant according to the visit }
$$


1 order of each ant in the taboo table, and update pheromone on the path according to the

2 following formula.

$$
\tau_{i j}(t+1)=(1-\delta) \tau_{i j}(t)+\sum_{k=1}^{m} \Delta \tau_{i j}{ }^{k}
$$

4 where $\delta$ represents the pheromone volatilization coefficient, which simulates the volatilization 5 of pheromone secreted by ants in nature over time. The value range of $\delta$ is ${ }^{(0,1)}$. If the value is 6 too large, the pheromone on the path will volatilize too fast, which will increase the possibility of 7 re-selecting the previously searched path, thereby greatly reducing the understanding space and 8 weakening the ability of algorithm to search globally. If the value is too small, although the 9 possibility of searching for global optimal solution is increased, it also weakens the convergence 10 of algorithm. $\Delta \tau_{i j}{ }^{k}$ represents the number of pheromone left by ant $k$ on the path $\langle i, j>$ in this 11 iteration.

12

\section{$13 \quad$ 3.2. Algorithm Design}

$14 \quad 3.2 .1$. Parameter selection optimization

15 In the realization of ant colony algorithm, there are many parameters that need to be initialized. 16 Pheromone and heuristic function, information quantity-heuristic function product, and 17 cooperative behavior between ants will seriously affect the convergence of algorithm [24]. At the 18 same time, the parameters of ant colony algorithm are also key factors affecting the efficiency of 19 its solution performance. Pheromone residual factor, information heuristic factor, expected 20 heuristic factor, pheromone intensity, number of ants, etc. are all very important parameters. The 21 selection method and selection principle directly affect the global convergence and solution 
1 efficiency of ant colony algorithm. The choice of different parameters can have a vital impact on

2 the performance of ant colony algorithm.

4 3.2.1.1. The influence of parameter $\alpha$ on the performance of ant colony algorithm

5 The information heuristic factor $\alpha$ reflects the relative importance of the amount of information

6 accumulated by ants in the process of movement in guiding the search of ant colony. The larger

7 the value, the more likely the ants will choose the path they have walked before, and the

8 randomness of the search will be weakened. When the value of the information heuristic factor

$9 \alpha$ is too small, the search of the ant colony is likely to fall into the local optimum prematurely.

10

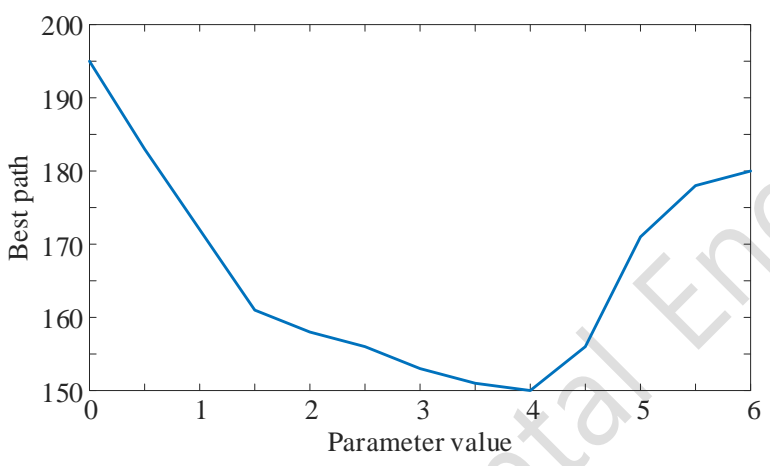

11 Fig. 2. Sensitivity analysis of parameter $\alpha$.

12 It can be seen from Fig. 2 that when the value of $\alpha$ is $1.5-4.5$, the comprehensive 13 solution performance of ant colony algorithm is better.

The expected heuristic factor $\beta$ reflects the relative importance of heuristic information in 17 guiding ant colony search process, and its size reflects the strength of a priori and deterministic factors in the ant colony optimization process. The larger the value is, the more likely the ant will 
1 choose the local shortest path at a certain local point. Although the convergence speed of 2 algorithm is accelerated at this time, the randomness of ant colony's search for the optimal path is 3 weakened, and it is easy to fall into the local optimal solution.

4

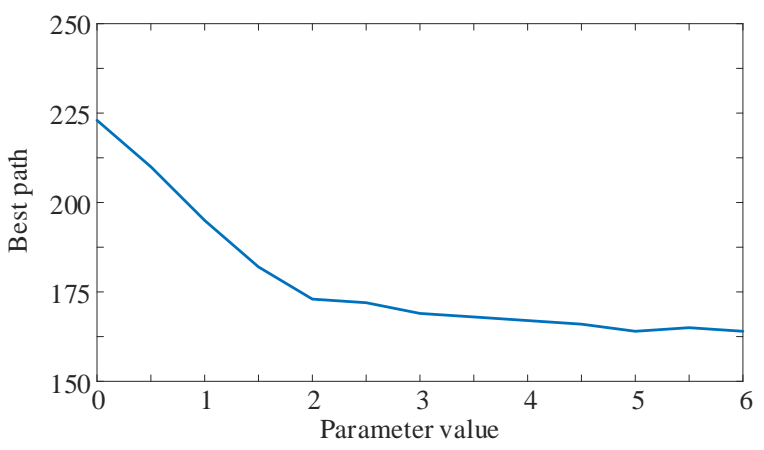

5 Fig. 3. Sensitivity analysis of parameter $\beta$.

It can be seen from Fig. 3 that when the parameter $\beta$ is $2-6$, the comprehensive solution

7 performance of ant colony algorithm is better.

$9 \quad 3.2 .1 .3$. The influence of parameter $\delta$ on the performance of ant colony algorithm

10 The size of the pheromone volatilization factor $\delta$ is directly related to the global search ability of 11 ant colony algorithm and its convergence speed. The pheromone residual factor ${ }^{(1-\delta)}$ reflects

12 the strength of individual interaction between ants. Due to the existence of the parameter $\delta$, 13 when the scale of the problem to be dealt with is relatively large, the amount of information on 14 the path that has never been searched will be reduced to close to 0 , thus affecting the global 15 search ability of algorithm. 


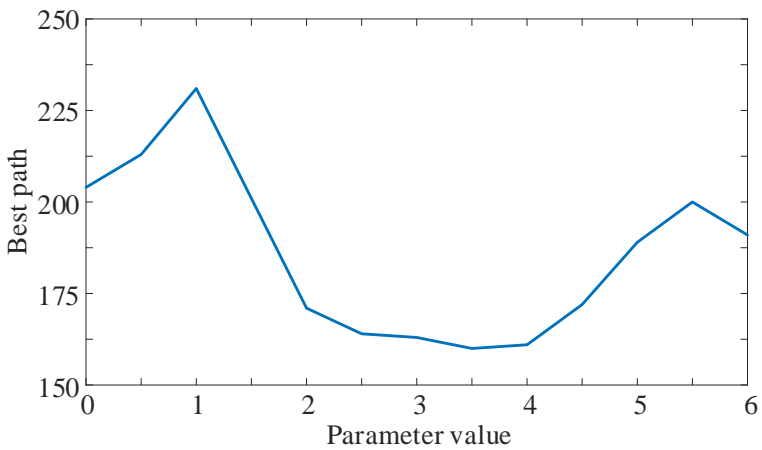

2 Fig. 4. Sensitivity analysis of parameter $\delta$.

3 It can be seen from Fig. 4 that under the same conditions of other initialization 4 parameters, when the pheromone residual factor is between 2 and 4 , the convergence 5 performance and convergence speed of ant colony algorithm are better, and the calculation 6 performance is also relatively stable.

8 3.2.1.4. The influence of parameter $\tau$ on the performance of ant colony algorithm

9 The pheromone intensity $\tau$ is the total amount of pheromone released on the path that the ant 10 circulates for a week. Its role is to make full use of the global information feedback on the 11 directed graph, so that the algorithm can search for the global optimal solution of problem at a 12 reasonable evolution speed under the action of positive feedback mechanism. When the Q value 13 is large, although the convergence speed of ant colony algorithm is very fast, the global search 14 ability of algorithm will deteriorate at this time, it is easy to fall into the local optimal solution, 15 and calculation performance becomes very unstable.

16 It can be known from experience that the model used in this paper is ant week model, and 17 the total amount of information has no obvious effect on the performance of ant colony 18 algorithm of ant week model. Generally take $\tau=100$. When the size of city becomes larger, the 
1 value of $\tau$ should be relatively increased, otherwise the total amount of information remains

2 unchanged. When the scale of the city is larger, the amount of information volatilized to each

3 path becomes smaller, and it is not easy to find the global optimal solution.

$4 \quad$ The model used in this paper is ant week model, and the total amount of information has

5 no obvious effect on the performance of ant colony algorithm of ant week model. Based on the

6 experimental analysis of parameter selection law for ant colony algorithm, the specific steps of

7 effective method for selecting the optimal combination parameters of ant colony algorithm are as

8 follows:

9 (1) Determine the number of ants, that is, the proposed selection strategy of city 10 size/number of ants $\approx 2$ to determine the total number of ants;

(2) Coarse parameter adjustment, that is, to adjust the information heuristic factor $\alpha$,

12 expected heuristic factor $\beta$, and pheromone intensity $\tau$ with a large value range to obtain a 13 more ideal solution;

(3) Parameter fine-tuning, that is, adjusting the pheromone volatilization factor $\delta$ with a 15 smaller value range.

16

17 3.2.2. Pheromone update strategy

18 When ${ }^{m}$ ants have completed a cycle, that is, when all ants have constructed a feasible path $R$, 19 compare it with the global optimal path $R^{*}$, where $R^{*}=\min \left(R_{k} \mid k=1,2, \ldots \ldots, m\right)$. 
1

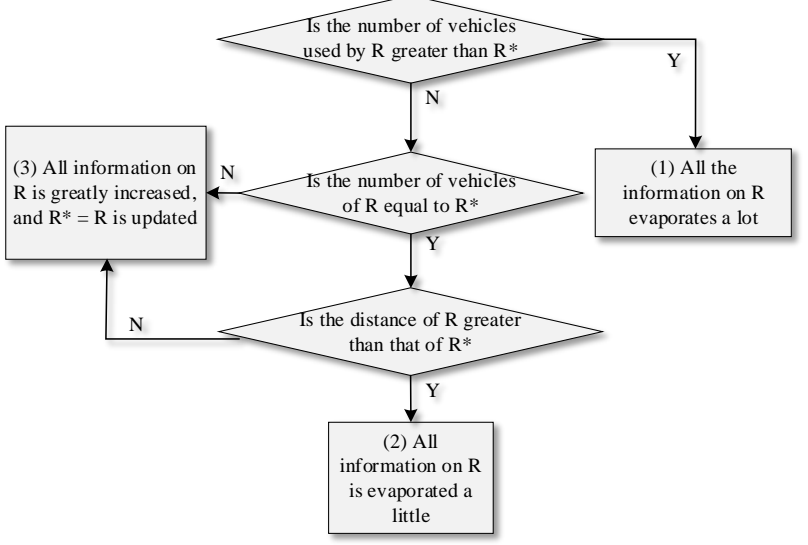

2 Fig. 5. Global pheromone update process.

3 In order to make each ant's iterative transportation scheme produce positive and negative

4 feedback on the increase and decrease of pheromone, according to the rule of Fig. 5, the

5 pheromone on all ants $R$ in each iteration is updated globally:

6 (1) The pheromone update formula is:

$$
\tau_{i j}^{\text {new }}=\theta^{2} \tau_{i j}^{\text {old }} \quad \forall(i, j) \in R
$$

8 (2) The pheromone update formula is:

$$
\tau_{i j}^{\text {new }}=\theta \tau_{i j}^{\text {old }} \quad \forall(i, j) \in R
$$

(3) The pheromone update formula is:

$$
\tau_{i j}^{\text {new }}=\max \left(\theta \tau_{i j}^{\text {old }}+\Delta \tau_{i j}{ }^{*}, \tau_{i j}^{\text {old }}\right)
$$

$$
\Delta \tau_{i j}^{*}=\frac{Q}{L^{*}} \quad(i, j) \in R
$$

$13 \quad Q$ is a constant, which represents the total amount of pheromone released by the ant to complete 14 a search; $L^{*}$ is the optimal path length explored by the ant. $\theta$ is the degree of pheromone 15 retention. In the early stage of algorithm, the diversity of understanding can take a relatively 
1 large value. In the middle and late stages, for the rapid convergence of the optimal solution, the

2 pheromone is more concentrated, and it can be smaller.

3 In order to avoid falling into a local optimal solution in the early stage, MMAS can be used for

4 reference to limit the pheromone within a certain range to avoid losing the possibility of 5 exploring new paths. which is

6

9 3.2.3. Optimize the optimal solution using neighborhood algorithm

In order to improve the convergence speed of algorithm, neighborhood algorithm is used to 11 optimize the feasible solution produced by each iteration of ant colony algorithm [25]. There are 12 three ways to find the neighborhood solution: insertion, reversal and exchange. In this paper, the 13 exchange method is used to find the neighborhood solution, that is, the solution produced by 14 each iteration of ant colony algorithm is first obtained. Then randomly select different delivery 15 points to exchange the positions of the two in the path where the solution is obtained, perform 16 random perturbation and recalculate to obtain a new solution. If the new solution is better than 17 the original solution, the solution is updated, otherwise it remains unchanged. Repeat the above 18 operations until the optimal solution is obtained when the maximum number of iterations is 19 reached.

20

\section{3.3. Algorithm Flow}


1 Step 1: Define the initial value of each algorithm parameter. The pheromone value on the road

2 section between any two points is initially $\tau_{\max }$, and ${ }^{N C_{-}} \max$ is set as the maximum number of

3 iterations of algorithm.

$4 \quad$ Step 2: Put $m$ ants in the parking lot, and put the point in the current solution, using the method

5 of constructing the solution in order.

6 Step 3: Calculate the transition probability, and require all constraints to be met at the same time,

7 select another demand point in addition to the current solution set, and then place it in the current

8 solution. If the next node that satisfies the constraint conditions is not found, return to the

9 parking lot.

10 Step 4: Cycle Step 3 until $m$ ants have visited all points, and get the corresponding loop that

11 starts from the parking lot and meets the constraints. The several loops of each ant are equivalent

12 to the distribution path formed by several transportation vehicles issued by vehicle site, and the

13 shortest path $R^{*}$ is calculated and saved.

14 Step 5: Perform global update and adjust $\theta$ according to the method described in this paper. The 15 adjustment rules are:

$$
\theta_{i}= \begin{cases}0.95 \theta_{t-1} & \text { if } \quad 0.95 \theta_{t-1} \geq \theta_{\min } \\ \theta_{\min } & \text { otherwise }\end{cases}
$$

17 Step6: Judge whether the number of iterations reaches the maximum value, if yes, terminate the 18 transportation, otherwise clear the taboo table and go to Step 2.

19

20

\section{Case Study and Verification}


1 The verification and simulation of the proposed method are all carried out on a high-performance

2 computer, so that the comparison method also runs in a unified simulation environment. The

3 experimental hardware and software configurations are shown in Table 1. The experimental

4 codes of the routing optimization method based on improved biological evolution algorithm are

5 all realized by Matlab language.

6

$7 \quad$ Table 1. Experimental Simulation Scenarios

\begin{tabular}{lll}
\hline \multirow{2}{*}{ Software environment } & Operating system & Windows10 \\
\cline { 2 - 3 } & Program editor & Matlab2016a \\
\hline \multirow{2}{*}{ Hardware environment } & CPU & $3.2 \mathrm{GHz}$ Intel Core i5-6500 CPU \\
\cline { 2 - 3 } & GPU & GeForce GTX 1050Max-Q \\
\cline { 2 - 3 } & Running memory & 8 GB
\end{tabular}

8

9 4.1. Network Parameters and Application Cases

10 In the improved ant colony routing optimization proposed method, the chromosome coding

11 uniformly adopts the natural number coding method. The parameter settings involved in ant

12 colony algorithm: $m=30, \alpha=2.5, \beta=3$ and $\delta=3$; the number of iterations is 300 as the

13 termination condition.

14 The experimental example uses an actual dataset in a certain area of North China, which

15 governs 31 villages and uses a waste vehicle with a maximum load of 7,000 kg. Table 2 shows

16 the serial number, name, coordinates and daily waste output of 1 depot, 31 villages, and a waste

17 treatment plant. 
1 Table 2. Actual Sample Dataset of a Certain Area

\begin{tabular}{|c|c|c|}
\hline No. & Name & Waste output $(\mathrm{kg})$ \\
\hline 1 & Parking lot & 0 \\
\hline 2 & Linglong goushang Village & 828 \\
\hline 3 & Shanqian Village & 517 \\
\hline 4 & Hengzhang Jiangjia Village & 925 \\
\hline 5 & Lugezhuang Village & 967 \\
\hline 6 & Mu Lin Zhuang Village & 840 \\
\hline 7 & Shengjia Village & 879 \\
\hline 8 & Wangjia Village & 872 \\
\hline 9 & Qianhuayuan Village & 696 \\
\hline 10 & Houhuayuan Village & 585 \\
\hline 11 & Panjiaji Village & 828 \\
\hline 12 & Oujiakuang Village & 853 \\
\hline 13 & Lugezhuang Village & 515 \\
\hline 14 & Lujia village & 638 \\
\hline 15 & Linglong Taishang Village & 523 \\
\hline 16 & Dajiangjia Village & 912 \\
\hline 17 & Xiaojiangjia Village & 548 \\
\hline 18 & Linglong Gaojia Village & 848 \\
\hline 19 & Fengjia Village & 658 \\
\hline 20 & Liujia Village & 776 \\
\hline 21 & Zhaizi Village & 517 \\
\hline 22 & Dongzhuangtou Village & 719 \\
\hline 23 & Xizhuangtou Village & 669 \\
\hline 24 & Dongtuanbu Village & 883 \\
\hline 25 & Xituanbu Village & 792 \\
\hline 26 & Huwangzhuang Village & 593 \\
\hline 27 & Longquanzhuang Village & 769 \\
\hline 28 & Gaojiatuan Village & 824 \\
\hline 29 & Guanjiahe Village & 775 \\
\hline 30 & Kuang Lujia village & 591 \\
\hline 31 & Lijia village & 628 \\
\hline 32 & Yuquanzhuang Village & 857 \\
\hline 33 & Transfer station & 0 \\
\hline
\end{tabular}

\section{$3 \quad 4.2$. Case Analysis}


1 In order to verify the feasibility and engineering applicability of the proposed method, we choose

2 reference [13], reference [14] and reference [16] as the comparison method, and our proposed

3 method is analyzed separately under a unified experimental scenario.

$4 \quad$ Fig. S1 is a schematic diagram of the optimal path proposed in this paper based on 5 improved ant colony algorithm. When the simulation experiment is about the 40th iteration, the 6 best path of waste transportation vehicles is:

Waste vehicle No. 1: 1-19-20-21-25-24-22-23-28-29-33-1

Waste vehicle No. 2: 1-2-3-9-10-12-17-16-15-18-31-33-1

Waste vehicle No. 3: 1-4-5-6-7-8-11-14-13-33-1

Waste vehicle No. 4: 1-26-27-30-32-33-1

The best path of garbage transportation vehicles and the lowest cost is shown in Figure S2. It can be seen from Figure S2 that when the number of iterations reaches 40, the improved ant colony algorithm can converge quickly, the proposed model tends to be stable, and the minimum cost of distribution after optimization is about 3,080 yuan.

At the same time, we also analyze the cost of carbon emissions. The carbon emissions under different methods are shown in Fig.S3.

Fig. S3 shows the carbon emissions under different methods. It can be seen from Fig. S3 that the proposed method in this paper achieves effective convergence in about 40 iterations, and the cost is roughly controlled at 3,080 yuan. Compared with reference [13] and reference [14], carbon emission costs are reduced by 100 and 70 yuan, respectively, and the convergence speed of comparison algorithm is significantly slower than our proposed method. However, within the specified number of iterations-300 times, the problem is still effectively solved, and it is still in a 
1 divergent state in the reference [16]. The reason is that this paper improves the pheromone

2 update strategy, which can realize the self-adaptive update of pheromone in the whole cycle of 3 solution. This limit avoids the routing optimization problem being vulnerable to the risk of local

4 optimization and realizes the optimization analysis of waste vehicle transportation path.

5

6

7

8

\section{1}

In addition, in order to more intuitively quantify the analysis performance of various methods, this paper presents the optimal path problem analysis capabilities under different methods in the form of Tab. S1. In Tab. S1, $G_{\text {best }}$ is the optimal value found, $G_{\text {avg }}$ is the average value, $P_{\text {best }}$ is the probability of finding the optimal solution, and $V_{a r}$ is the average deviation.

As shown in Tab. S1, when the waste vehicle transportation path is optimally solved, as the solution performance stability index, the value of our proposed method is 0.87 , which is an improvement of 0.08 compared to the reference [14]. The average deviation value of the indicators analyzed in this paper is 0.011 , which is 0.03 lower than that in the reference [14]. This shows that the proposed method can effectively analyze complex path analysis problems under the same experimental conditions. The comparison of indicators GB and G illustrates this phenomenon. The indicator values obtained by the proposed method are significantly lower than the comparison method.

In summary, this paper proposes a path analysis method for waste vehicle transportation based on improved biological evolutionary algorithm. This method is oriented to low carbon and environmentally-friendly transportation requirements, which can realize efficient and accurate optimal routing optimization analysis.

\section{Conclusions}


1 Aiming at the low efficiency of current VRP problem analysis, this paper considers the concept

2 of low carbon environmental protection and proposes a new method for optimizing waste

3 transportation vehicles based on improved ant colony algorithm. This method improves

4 pheromone by combining and optimizing network parameters, and realizes the full-cycle

5 adaptive update of algorithm. Moreover, it introduces neighborhood algorithm to iteratively

6 optimize the biological evolution algorithm, which further improves the solution performance of

7 VRP algorithm. Experimental results show that the proposed method can achieve optimal

8 solution of complex paths, and it has better execution efficiency than other methods. The future

9 research direction will make VRP problem more concrete and study the routing optimization

10 method of waste transportation vehicles under fuzzy demands. In addition, due to the high

11 timeliness requirements of waste transportation, the driving time in the distribution process will

12 generally be affected by factors such as weather conditions, road conditions, emergency time and

13 vehicle status. Therefore, the uncertainty of these factors can be more considered in the future

14 research on waste transportation.

\section{Author Contributions}

17 The main idea of this paper is proposed by F.L.S. (engineer). All the work of the article was 18 completed by F.L.S. himself. 
1 [1] Elgarej M, Mansouri K, Youssfi M, Nezha B, Fazazi EL. Distributed Swarm

2 Optimization Modeling for Waste Collection Vehicle Routing Problem. Int. J. Adv. Comput.

$3 \quad$ SC. 2017;8(9):306-312.

4 [2] Vu HL, Bolingbroke D, Ng KTW. Assessment of waste characteristics and their impact

5 on GIS vehicle collection route optimization using ANN waste forecasts. Waste. Manage.

$6 \quad 2019 ; 88(1): 118-130$.

7 [3] Rybnytska O, Burstein F, Rybin AV. Decision support for optimizing waste management.

$8 \quad$ J. Decis. Syst. 2018;27(1):68-78.

9 [4] Zhang SQ, Mu D, Wang C. A Solution for the Full-load Collection Vehicle Routing 10 Problem with Multiple Trips and Demands: An Application in Beijing. IEEE. Access. $11 \quad 2020 ; 8(1): 89381-89394$.

12 [5] Codu MK, Yilmaz M, Codur MY. Arc Routing Problem Approach for Reducing Exhaust 13 Gas Emission in Road Transportation: A Case Study of Erzurum. Fresen. Environ. Bull. $14 \quad 2019 ; 28(10): 7196-7205$.

15 [6] Benrahou F, Tair A. Capacitated Vehicle Routing Problem for Collection Waste Lube Oil 16 in Algiers. Fresen. Environ. Bull. 2019;28(6):4500-4505.

17 [7] Banyai T, Tamas P, Illes B, Živilè S, Ágota B. Optimization of Municipal Waste 18 Collection Routing: Impact of Industry 4.0 Technologies on Environmental Awareness and 19 Sustainability. Int. J. Envir. Res. Pub. HE. 2019;16(4):1-26.

20 [8] Zhao HX, Liu GS, Li Y. Optimal path planning for classified waste recovery based on 21 random walk. J. Transp. Eng. Inf. 2018;16(3):103-108. 
1 [9] $\mathrm{Vu} \mathrm{HL}, \mathrm{Ng} \mathrm{K}$, Fallah B, Richter A, Kabir G. Interactions of residential waste

2 composition and collection truck compartment design on GIS route optimization. Waste.

$3 \quad$ Manage. 2019;102(1):613-623.

4 [10] Wohlk S, Laporte G. A districting-based heuristic for the coordinated capacitated arc 5 routing problem. Comput. Oper. Res. 2019;111(1):271-284.

6 [11] Yang J, Qiu K. Routing Optimization of White Pollution Garbage Cleaning and

7 Transportation Vehicles using MMKCA Algorithm under the Perspective of Low Carbon and

8 Environmental Protection. Fresen. Environ. Bull. 2021;30(4):4402-4410.

9 [12] Amal L, Le HS, Chabchoub H., Lahiani H. Analysis of municipal solid waste collection 10 using GIS and multi-criteria decision aid. Appl. Geomat. 2019;12:193-208.

11 [13] Ge XL, Zhang H. Research on vehicle routing optimization of urban real-time traffic 12 network. Ind. Eng. Manage. 2018;23:140-149.

13 [14] Li ZP, Zhang YW. Multi demand vehicle routing problem with time windows and service 14 sequence constraints. Control. Decis. 2019;34:1565-1570.

15 [15] Bansal S, Wadhawan S. A Hybrid of Sine Cosine and Particle Swarm Optimization 16 (HSPS) for Solving Heterogeneous Fixed Fleet Vehicle Routing Problem. Int. J. Appl. 17 Metaheur. 2021;12:41-65.

18 [16] Huang GW, Cai YG. Adaptive genetic gray wolf optimization algorithm for vehicle 19 routing problem with capacity constraint. Acta. Electron. Sinica. 2019;47:2602-2610.

20 [17] Rossit DG, Nesmachnow S, Toutouh J. A bi-objective integer programming model for 21 locating garbage accumulation points a case study. Rev. Fac. Ing. 2019;93:70-81. 
1 [18] Shen Y, Liu M, Yang J, Shi Y, Middendorf M. A Hybrid Swarm Intelligence Algorithm

2 for Vehicle Routing Problem with Time Windows. IEEE. Access. 2020;8:93882-93893.

3 [19] Guo YN, Cheng J, Luo S, Gong DW. Robust Dynamic Multi-objective Vehicle Routing

4 Optimization Method. IEEE. ACM. T. Comput. BI. 2018;15:1891-1903.

5 [20] Vega-Mejía CA, Montoya-Torres JR, Islam S. A nonlinear optimization model for the

6 balanced vehicle routing problem with loading constraints. Int. T. Oper. Res. 2019;26:794-835.

7 [21] Saeheaw T, Charoenchai N. A comparative study among different parallel hybrid

8 artificial intelligent approaches to solve the capacitated vehicle routing problem. Int. J. Bio-

$9 \quad$ Inspir. Com. 2018;11:171-191.

10 [22] Zhang S, Gajpal Y, Appadoo SS. A meta-heuristic for capacitated green vehicle routing 11 problem. Ann. Oper. Res. 2018;269:753-771.

12 [23] Wu Y, Pan F, Li S, Chen Z, Dong M. Peer-induced fairness capacitated vehicle routing 13 scheduling using a hybrid optimization ACO-VNS algorithm. Soft. Comput. 2019;24:2201142213.

15 [24] Huang YH, Blazquez CA, Huang SH, Paredes-Belmar G, Latorre-Nuñez G. Solving the 16 Feeder Vehicle Routing Problem using ant colony optimization. Comput. Ind. Eng. $17 \quad 2019 ; 127: 520-535$.

18 [25] Wang JH, Weng TY, Zhang QF. A Two-Stage Multiobjective Evolutionary Algorithm 19 for Multiobjective Multidepot Vehicle Routing Problem With Time Windows. IEEE. T. 20 Cybernetics. 2019;49:2467-2478. 